\title{
QUANTUM MECHANICAL TUNNELLING AND ITS APPLICATIONS
}


This page is intentionally left blank 


\title{
Quantum Mechanical Tunnelling And Its Applications
}

\author{
D.K. ROY* \\ DEPARTMENT OF PHYSICS \\ INDIAN INSTITUTE OF TECHNOLOGY, DELHI \\ NEW DELHI 110016, INDIA
}

*Resident Visitor

AT\&T Bell Laboratories

Murray Hill, New Jersey 07974

U.S.A 
Published by

World Scientific Publishing Co Pte Ltd

P. O. Box 128, Farrer Road, Singapore 9128.

242 Cherry Street, Philadelphia PA 19106-1906, USA.

\section{Library of Congress Cataloging-in-Publication Data}

Roy, D. K.

Quantum mechanical tunnelling and its applications.

Bibliography. p.

1. Tunnelling (Physics) I. Title.

QC176.8.T8R69 $1986 \quad 530.4 '$

ISBN 9971-50-024-8

86-22446

Copyright $\odot 1986$ by World Scientific Publishing Co Pte Ltd.

All rights reserved. This book, or parts thereof, may not be reproduced in any form or by any means, electronic or mechanical, including photocopying, recording or any information storage and retrieval system now known or to be invented, without written permission from the Publisher.

Printed in Singapore by Kyodo-Shing Loong Printing Industries Pte Ltd. 


\section{PREFACE}

The concept of Quantum Mechanical Tunnelling appeared to emerge as one of the immediate consequences of wave mechanics. Once, a propagating stream of material particles is represented by its wave function, according to the conventional analyses, the former is able to penetrate a reasonably thin barrier without any real tunnel existing across it even when its energy remains far smaller than the barrier height. Several physical phenomena e.g. alpha decay, high field electron emission from cold metals, auto-ionization of hydrogen atoms etc. were reported to be explained on the basis of the abovementioned idea.

The study of Quantum Mechanical Tunnelling through solid-state junctions, however, became an area of intense research activity with the discoveries of Esaki and Josephson effects because of their innumerable applications. The inabilities of the conventional tunnelling theories to explain adequately the current flow through such junctions have exposed our limitations of truly understanding the physical basis of the process of quantum mechanical tunnelling itself. The primary aim of this work is to present a new theoretical account of the latter based on the principle of Quantum Measurement and Observation. The essence of this theory is that during tunnelling transition an electron acquires a state intermediate to that of a matter and a wave when it is physically impossible to assign its energy until the process of tunnelling is over. After having presented a brief review of the conventional tunnelling theories in Chapter I, the new tunnelling theory has been developed, in detail, in Chapter II where its basic parameters, such as the tunnelling probability, the tunnelling time and the tunnelling current density are derived so as to be applicable to real problems of interest. Chapters III and IV, on the other hand, review the physics of solid state devices involving rectangular and triangular barrier systems from the viewpoint of the new tunnelling theory. Chapter V, in the end, presents some of the topics whose adequate understanding is a prerequisite to proper appreciation of the contents of the text. 
An earlier edition of the work entitled, "Tunnelling and Negative Resistance Phenomena in Semiconductors" appeared from Pergamon Press, Oxford, England in 1977. However, the difference of the present work from its predecessor should be mentioned at the outset. This edition contains an up-to-date account of our work on Tunnelling which was just initiated earlier and includes a derivation of Josephson Effect from the standpoint of the new theory. The discussion on negative resistance microwave devices has now been excluded. The emphasis is presently more on the development of physical principles of operations of solid-state devices rather than on the discussion of their technology of fabrication and circuit applications. An elementary understanding of wave mechanics and physics of semiconductor devices should enable the readers of the book to fully appreciate its contents. The book has primarily been intended to be a work of reference to post-graduate, doctoral and post-doctoral students along with research workers working in the area of solid-state devices. This essentially is an outcome of my teaching and research experiences at the Indian Institute of Technology, Delhi, India for nearly two decades. The efforts of my doctoral students viz. P. J. George, P. N. Roy, P. N. Singh, R. Y. Thakur, K. N. Rai, S. K. Ghosh, N. S. T. Sai, Amitabh Ghosh and G. Tiwary in contributing towards the development of some of the contents of this work are worth mentioning.

In the course of writing this book I have received assistance from several quarters. Firstly, I must acknowledge with thanks the authorities of the Rotary Foundation of the Rotary International for granting me a fellowship to visit AT\&T Bell Laboratories at Murray Hill during 1986. Secondly, the authorities of the Indian Institute of Technology, Delhi deserve admiration for promptly granting me a sabbatical leave to come over to USA. Finally, I must thank the management of the Bell Laboratories for providing me a challenging environment and all the secretarial assistance necessary for the satisfactory completion of this work. Efficient typing of the manuscript by Anna $\mathrm{H}$. Jacobsen is hereby being thankfully acknowledged. Thanks are also due to Federico Capasso, Gene Baraff and several other members of the Technical Staff of Bell Laboratories for a number of stimulating discussions that I had with them while writing this book. Special thanks are due to V. Narayanamurti, Director of Solid-State Electronics Research for 
welcoming me and making everything possible to make my stay here comfortable and enjoyable. Finally, I wish to express great appreciation for the gestures of my wife Tripti Roy, my daughter Mini and my son Bobby for leaving me alone to come to Bell Laboratories to accept this assignment. 
This page is intentionally left blank 


\section{CONTENTS}

Preface

\section{Chapter I: Quantum Mechanical Tunnelling}

1.1 Introduction 2

1.2 Conventional Formulations of the Tunnelling Problem 4

1.2.1 Time-independent Approach 4

1.2.2 Time-dependent Perturbation Approach 19

1.2.3 Equivalence of Time-independent and

Time-dependent Perturbation Approaches 22

1.3 Inadequacies of Conventional Tunnelling Formulations 24

1.4 A Recent Application of Tunnelling 31

1.5 Summary 32

Chapter II: The New Tunnelling Theory

2.1 The Barrier Hamiltonian and the Barrier Wavefunction 36

2.2 The Tunnelling Probability and the Tunnelling Time 49

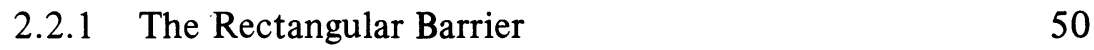

2.2.2 The Triangular Barrier $\quad 56$

2.3 The Tunnelling Current Density 66

$\begin{array}{ll}\text { 2.3.1 The Rectangular Barrier } & 67\end{array}$

$\begin{array}{ll}\text { 2.3.2 The Triangular Barrier } & 75\end{array}$

2.4 Indirect Tunnelling $\quad 78$

2.4.1 Barrier Hamiltonian and Wave Function 79

2.4.2 The Tunnelling Probability and the Tunnelling Current Density $\quad 88$

2.5 Summary

93

Chapter III: Tunnelling in Rectangular Barrier Systems

3.1 Metal-Insulator-Metal (MIM) Junction 99

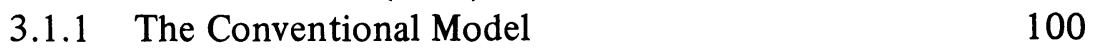

3.1.2 Tunnelling Characteristics on New Tunnelling Theory

3.1.3 Practical Applications of MIM Junctions 
3.2 Metal-Insulator-Semiconductor (MIS) System 106

3.2.1 Metal-Insulator-Semiconductor

(Nondegenerate) Junction

106

3.2.2 MIS Junction with Degenerate Semiconductor

Substrates

121

3.3 Metal-Insulator-Semiconductor Switch (MISS)

124

3.4 Normal Metal-Insulator-Superconductor (NIS) Junction

129

3.5 Superconductor-Insulator-Superconductor $\left(\mathrm{S}_{1}-\mathrm{I}-\mathrm{S}_{2}\right)$

Junction

133

3.6 Josephson Effect

3.6.1 D.C. Josephson Effect

3.6.2 A.C. Josephson Effect

3.6.3 The Effect of Microwave Power on a Josephson Junction

3.6.4 Effect of Magnetic Field on Josephson Current

3.6.5 Josephson Quantum Phase Detector

3.6.6 Application of Josephson Effect

3.6.7 Inadequacies of the Conventional Explanation of Josephson Effect

3.6.8 Explanation of Josephson Effect on the New Theory

3.6.9 The Temperature Dependence of $\mathrm{J}_{01}$ and $\mathrm{J}_{02}$

3.6.10 The Effect of the Magnetic Field on the Pair Tunnelling Current

3.7 The Semiconductor Heterostructures

3.7.1 The Negative Differential Conductivity Effects

A. Bloch Oscillation Model

B. Resonant Transmission Across Double Barriers

C. Transport Across an Array of One-Dimensional Barriers

3.7.2 Modulation Doping of Superlattices

3.7.3 The Quantum Hall Effect

3.7.4 Semiconductor-Semimetal Transition in Superlattices

3.7.5 Miscellaneous Other Effects with Superlattices 190

A. Shubnikov-de Haas (SdH) Effect 190

B. Magnetophonon Effect

C. Infrared Detection

D. Tailoring of Potential Profiles 
E. Superlattice Avalanche Photodiode 192

F. Strained-layer Heteroepitaxy 192

3.8 Summary

193

Chapter IV: Tunnelling in Triangular Barrier Systems

4.1 Metal-Vacuum Boundary 197

4.1.1 Field Emission 199

4.2 Metal-Semiconductor Contact 203

4.2.1 The Barrier Structure 204

A. Ideal Case (no surface state) 205

B. Imperfect Case (large density of surface states) 208

C. The Shape of a Schottky Barrier 210

4.2.2 Current Transport Across a Schottky Barrier 212

A. Thermionic Emission Theory 212

B. Diffusion Theory 215

C. Tunnelling Theory $\quad 217$

4.3 Semiconductor $p n$ Junctions 218

4.3.1 Zener Diodes 218

4.3.2 Backward Diodes 226

4.3.3 Tunnel Diodes 227

A. A Qualitative Explanation of the Device I-V
Characteristics

B. Theoretical Derivation of I-V Characteristics on the Basis of the Conventional Model 234

C. Derivation of I-V Curve on New Tunnelling Concepts 243

D. Indirect Tunnelling Current 247

E. Excess Current 253

F. Thermal Current in Tunnel Diodes 259

G. Prediction of Tunnel Diode I-V Characteristics 262

H. Dependence of Tunnel Characteristics on Various Parameters 263

4.3.4 Circuit Applications of Tunnel Diodes 266

A. Equivalent Circuit and Stability Considerations 266

B. Tunnel Diode Amplifiers 272

C. Tunnel Diode Oscillators 274

D. Tunnel Diode Mixers, Converters and Detectors 275

E. Digital Applications 278 
4.4 Semiconductor Heterojunctions 280

4.4.1 Theoretical Models 280

A. Diffusion Model 281

B. Thermionic Emission Model 286

C. Tunnelling Model 287

4.4.2 Difficulties with Anderson's Model 287

4.4.3 Attempts to Remedy 288

A. The Model of Adams and Nussbaum 288

B. A New Proposal 290

4.5 Super-Schottky Junction $\quad 292$

4.6 Summary 294

Chapter V: Appendix

A.1 Airy Function and its Properties 296

A.2 Heavy Doping Effects 306

A.2.1 Mott Transition $\quad 310$

A.2.2 Impurity Ionization Energy ... 313

A.2.3 Electron Energy Spectrum 315

A.2.4 The Position of Fermi Level in Heavily Doped Semiconductors 323

A.2.5 The Percentage of Ionization of Carriers 326

$\begin{array}{ll}\text { A.3 Superconductivity } & 328\end{array}$

A.3.1 Phenomenological Theories on Superconductivity $\quad 330$

A.3.2 Microscopic Theory of Superconductivity 338

A.4 Quantum Transport Phenomena $\quad 347$

A.4.1 Magnetic Quantization , $\quad 347$

A.4.2 Electric Quantization / 353

A.5 Principle of Quantum Measurements and Observations 355

References 